\title{
Forward hadron calorimeter (PSD) of the NA61/SHINE detector for heavy ion studies and its upgrade for experiments beyond 2020
}

\author{
Sergey Morozov ${ }^{1}$ for the NA61/SHINE collaboration \\ Institute for Nuclear Research, Russian Academy of Sciences, Moscow, Russia \\ National Research Nuclear University MEPhI, Moscow, Russia \\ E-mail: morozovseinr.ru
}

\section{Fedor Guber}

Institute for Nuclear Research, Russian Academy of Sciences

E-mail: gubereinr.ru

\section{Alexander Ivashkin \\ Institute for Nuclear Research, Russian Academy of Sciences \\ E-mail: ivashkindinr.ru \\ Marina Golubeva \\ Institute for Nuclear Research, Russian Academy of Sciences \\ E-mail: marinaeinr.ru}

\begin{abstract}
The Projectile Spectator Detector (PSD) is a segmented modular hadron calorimeter currently used in the NA61/SHINE experiment at the CERN SPS. The PSD is used for collision centrality determination as well as for estimation of the event plane orientation in nucleus-nucleus collisions. The main goal of the NA61/SHINE experiment includes studying the onset of deconfinement and searching for the critical point of strongly interacting matter. It is of crucial importance to have a precise event class characterization for the analysis of event-by-event observables. The PSD has already been used for centrality selection at the trigger level in measurements of $\mathrm{Be}+\mathrm{Be}, \mathrm{Ar}+\mathrm{Sc}, \mathrm{Xe}+\mathrm{La}$ and $\mathrm{Pb}+\mathrm{Pb}$ reactions at a wide range of beam energies $(13-158 \mathrm{AGeV})$. The performance of the PSD in different collision systems will be shown and the proposed upgrade of the forward hadron calorimeter system for future experiments planned at NA61/SHINE beyound 2020 will be presented.
\end{abstract}

Corfu Summer Institute 2018 "School and Workshops on Elementary Particle Physics and Gravity" (CORFU2018)

31 August - 28 September, 2018

Corfu, Greece

\footnotetext{
${ }^{1}$ Speaker
} 


\section{Introduction}

NA61/SHINE is the fixed target experiment at the CERN SPS (Super Proton Synchrotron) pursuing a wide range of investigations in strong interactions as well as performing measurements for neutrino and cosmic rays studies. The main goal of strong interaction program at NA61/SHINE is to study strongly interacting matter namely search for the critical point $(\mathrm{CP})$ and investigate the properties of the onset of deconfinement. For these purposes a two-dimensional scan was performed in beam momenta (13A-150A/158A GeV/c) and size of colliding systems $(\mathrm{p}+\mathrm{p}, \mathrm{p}+\mathrm{Pb}, \mathrm{Be}+\mathrm{Be}, \mathrm{Ar}+\mathrm{Sc}, \mathrm{Xe}+\mathrm{La}, \mathrm{Pb}+\mathrm{Pb})$. The onset of deconfinement is studied in collisions of small and medium size nuclei [1]. The NA61/SHINE strong interaction program has been extended to $\mathrm{Pb}+\mathrm{Pb}$ interactions in order to obtain first measurements of open charm production in heavy ion collisions at SPS energies. In addition, anisotropic flow effects, as well as fluctuations in $\mathrm{Pb}+\mathrm{Pb}$ collisions are being explored. The systems already studied and those planned to be recorded in the future are shown in Fig. 1. The box sizes are proportional to the number of events recorded.

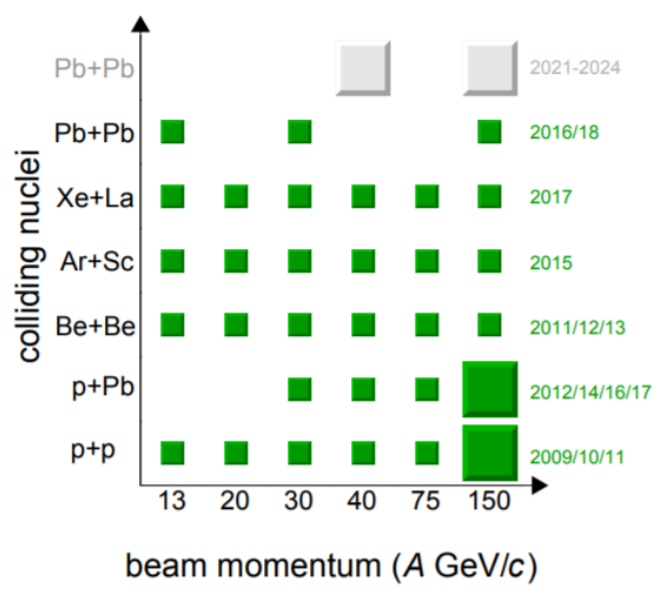

Fig.1. Strong interaction program of NA61/SHINE experiment. Data recorded (green) and planned (gray).

A variety of observables is measured in relativistic heavy ion collisions in order to investigate properties of hot dense matter in the collision zone. These are particle yields, transverse momentum spectra, rapidity and angular distributions. In addition, collective flows studies require event-by-event reaction plane angle reconstruction and fluctuation analysis needs information about the centrality of ion-ion collision. For such measurements forward hadron calorimeters are used in heavy ion experiments. This article presents the performance of the hadron sampling lead/scintillator calorimeter of the NA61/SHINE experiment at the CERN SPS. A future upgrade due to new requirements is discussed as well. 


\subsection{Forward sampling hadron calorimeter (PSD - Projectile Spectator Detector) for NA61/SHINE experiment.}

A schematic view of the NA61/SHINE experiment is shown in Fig.2 [2]. The main detectors are five time projection chambers (TPC): two vertex TPCs mounted in the magnets, a gap TPC covering the beam area and two main TPCs (left and right) measuring tracks of charged secondary particles. A TOF system for mass measurement and Projectile Spectator Detector (PSD) are placed downstream. In addition there is beam line instrumentation used in event selection and trigger generation. The NA61/SHINE track and vertex reconstruction procedures are discussed in Refs. [3,4,5].

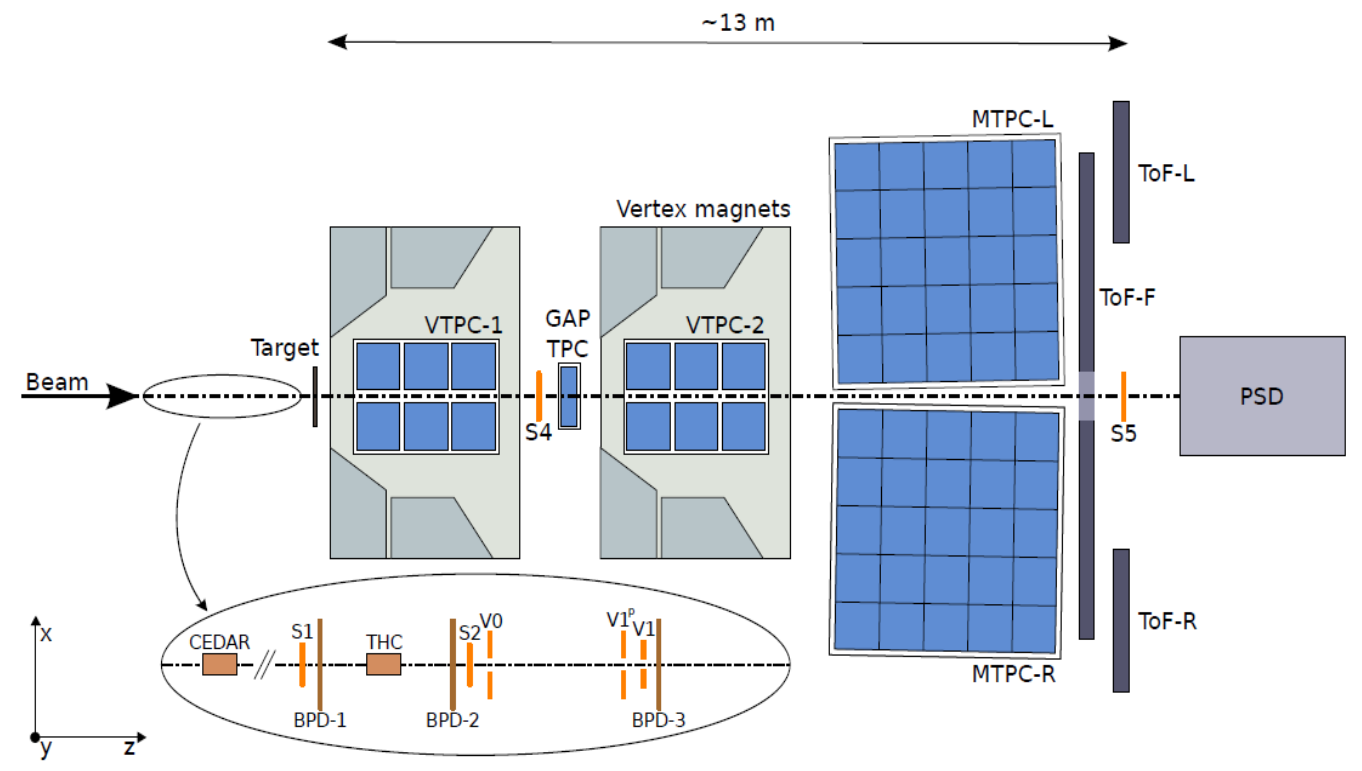

Fig.2. Schematic view of the NA61/SHINE experiment facility.

The Projectile Spectator Detector (PSD) is a sampling hadron calorimeter with modular structure. A photo of the PSD installed in the NA61/SHINE experimental area is shown in Fig.3 (left). A schematic view of the module structure is presented in Fig.3 (right). Each module has 60 lead/scintillator "sandwiches" composed of $16 \mathrm{~mm}$ thick lead plates and $4 \mathrm{~mm}$ thick scintillation plastic plates. This ratio (4:1) provides a compensating condition for hadronic shower measurements. Six consecutive layers of lead/scintillator form one section in the module. Light signals are read out with one photo-detector for each section through wavelength shifting fibers (WLS) glued into grooves in the scintillation plates. One module has 10 sections which provides the possibility to obtain longitudinal hadron shower information and to reduce the required dynamic range of the photo-detectors by sharing the light from the shower between several sections. To read the light Zekotek multipixel avalanche photo-diodes (MAPD) as well as modern Hamamatsu milti-pixel photon counters (MPPC) are used. The central part of the PSD consists of 16 modules with $10 \mathrm{~cm} \times 10 \mathrm{~cm}$ transverse size and the outer region has modules with $20 \mathrm{~cm} \times 20 \mathrm{~cm}$ size. The length of the PSD is about $1.2 \mathrm{~m}$ corresponding to $\sim 5.6$ interaction lengths. In order to have longer depth for high energy hadron showers a short 
module with 2 sections only has been constructed and installed in front of the PSD face. It covers the place of intersection of four small modules in the very center of the calorimeter. Nonperfect fit of modules in the center led to shower leakage especially for heavy ions and introduced a bias in the trigger signal derived from the 16 small modules of the calorimeter. The short module spreads the shower from ions among the four very central small modules reducing the impact of the huge light signal generated from heavy ion hadron showers.
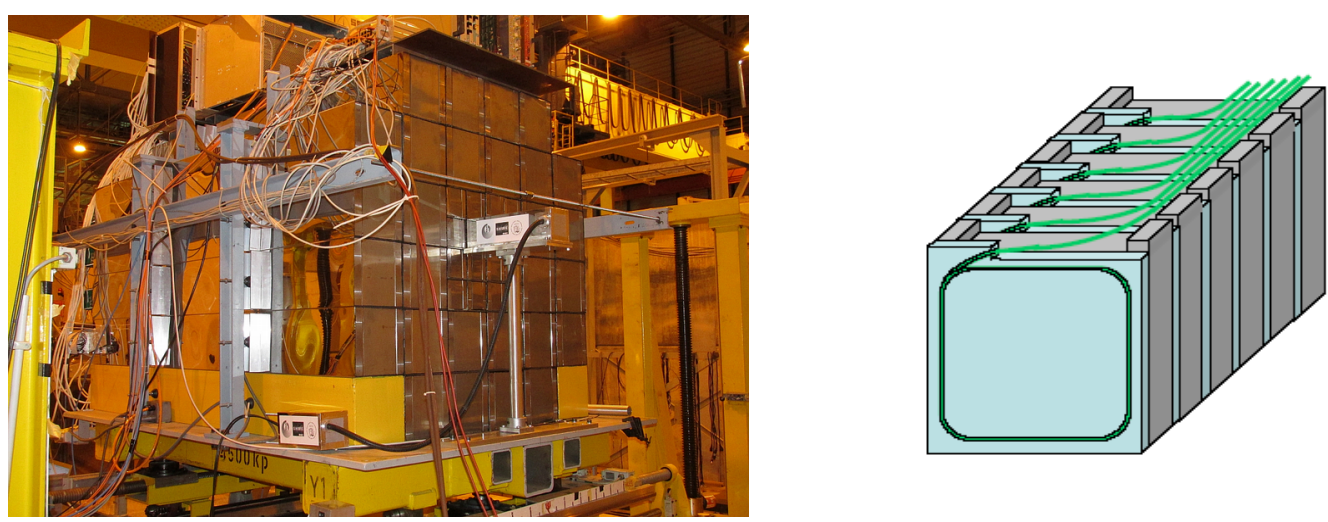

Fig.3. View of the PSD installed in the NA61/SHINE beam line (left) and schematic view of one PSD section with lead/scintillator "sandwich" structure (right).

\subsection{Calibration of PSD hadron calorimeter and single particle response.}

The main aim of forward hadron calorimeters is energy measurement of non-interacting nucleons (projectile spectators) in ion-ion collisions. The number of interacting participants $\mathrm{N}_{\mathrm{P}}$ can be calculated as:

$$
N_{p}=A-\frac{E_{s}}{E_{a}}
$$

where $\mathrm{A}$ is the mass number of the ion, $\mathrm{E}_{a}$ is the beam energy per one nucleon and $\mathrm{E}_{\mathrm{S}}$ is the energy carried by the non-interacting nucleons. In order to estimate $\mathrm{E}_{\mathrm{S}}$ correctly the calorimeter has to be calibrated. The calibration is done in two steps: using the most probable value (MPV) of the muon signal distribution for all sections to equalize their responses and the proton energy scan to determine the conversion factor from visible energy to full energy of the hadron shower. The homogeneity response scan w.r.t. position of the proton at front face of the PSD was performed during calibration by moving the calorimeter on its platform and consecutively exposing of each module one by one. The result of such a scan of modules is shown in Fig. 4 (left). The energy resolutions obtained are presented in Fig.4 (right). Fluctuations of energy responses are caused by non-identical design of modules as well as differences in electronic readout channels. Each module then has an additional individual calibration constant which was iteratively calculated to cancel the module-by-module response variations. Spectra from the proton energy scan from 20 to $158 \mathrm{GeV} / \mathrm{c}$ beam momenta are shown in Fig.5 (left). The linearity of response is shown in Fig.5 (right). Proton spectra for low energies have Gaussian shape while for high energies a tail is visible on the left side. This tail is caused by leakage of the hadronic 
showers through the back side of the calorimeter. For high energy ions this leakage has to be taken into account because it can lead to some bias in collision centrality determination. One can see from Fig.5 (left) that up to $80 \mathrm{GeV} / \mathrm{c}$ particle momentum the leakage is almost not visible in single protons but for large systems like $\mathrm{Xe} \mathrm{or} \mathrm{Pb}$ the leakage is not negligible again because of statistics.
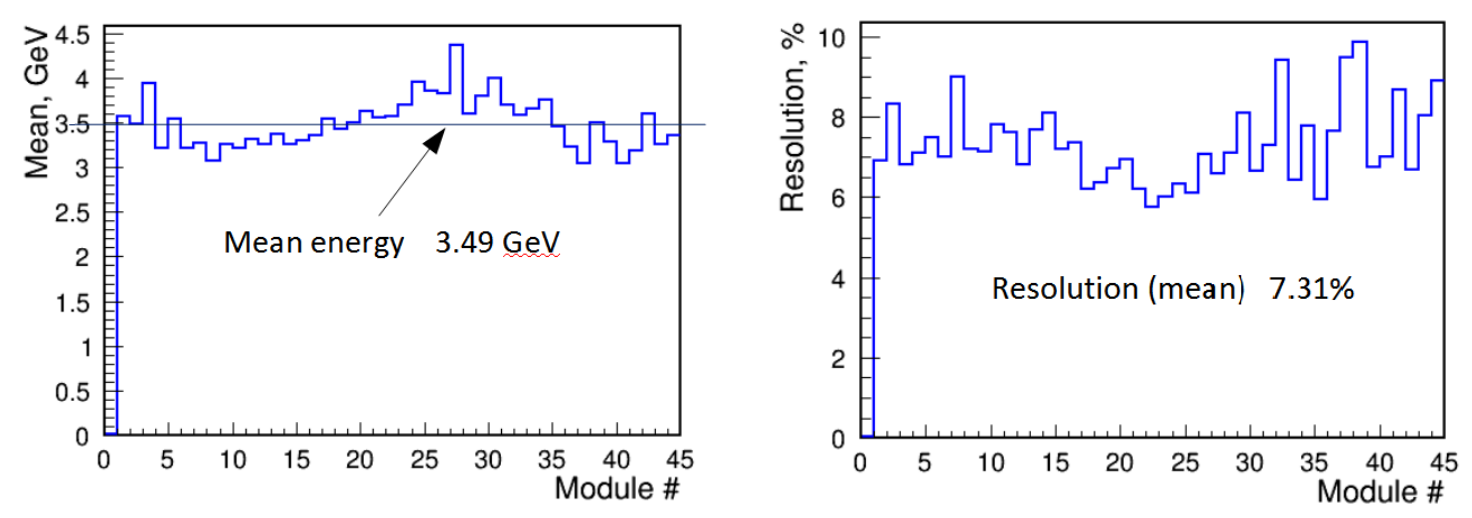

Fig.4. Result of module-by-module scan with $158 \mathrm{GeV} / \mathrm{c}$ momentum protons. Variations of visible energy in the calorimeter (left) and of energy resolution (right) are shown.
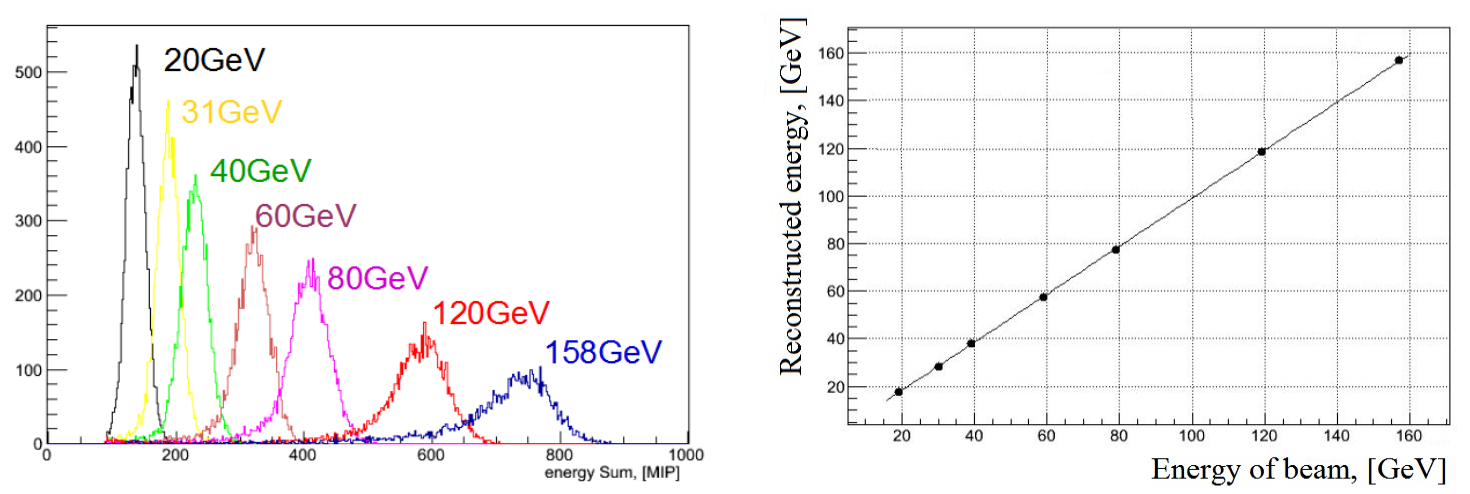

Fig.5. Proton calibration spectra (left) and linearity of calorimeter response (right).
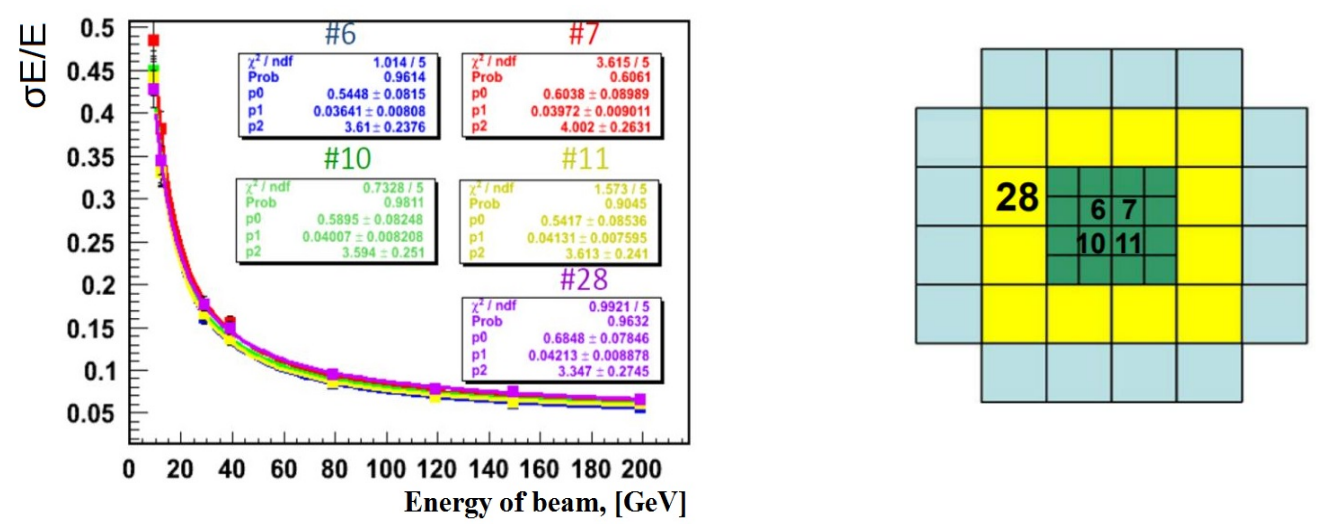

Fig.6. Proton calibration energy resolution curves (left) obtained from the energy scan with the beam centered on various modules of the calorimeter (see picture on right). 
In order to quantify the performance of the hadron calorimeter the resolution curve (see Fig.6 left) was fitted with the function:

$$
\frac{\sigma_{E}}{E}=\sqrt{\left(\frac{a}{\sqrt{E}}\right)^{2}+b^{2}+\left(\frac{c}{E}\right)^{2}}
$$

where $\mathrm{a}, \mathrm{b}$ and $\mathrm{c}$ are stochastic, constant and noise terms of hadronic energy resolution, respectively. The fitted values of the parameters are: $\mathrm{a}=56.8 \%, \mathrm{~b}=3.9 \%$ and $\mathrm{c}=3.8 \mathrm{GeV}$ for the beam centered on the small modules and $\mathrm{a}=68.5 \%, \mathrm{~b}=4.2 \%$ and $\mathrm{c}=3.3 \mathrm{GeV}$ for beam on the large module.

\section{Performance of the PSD hadron calorimeter of NA61/SHINE in ion-ion collisions.}

The ion-ion physics program of NA61/SHINE started with Be+Be collisions at energies of 40, 75 and $150 \mathrm{AGeV}$ where the performance of the just completed PSD with the first version of
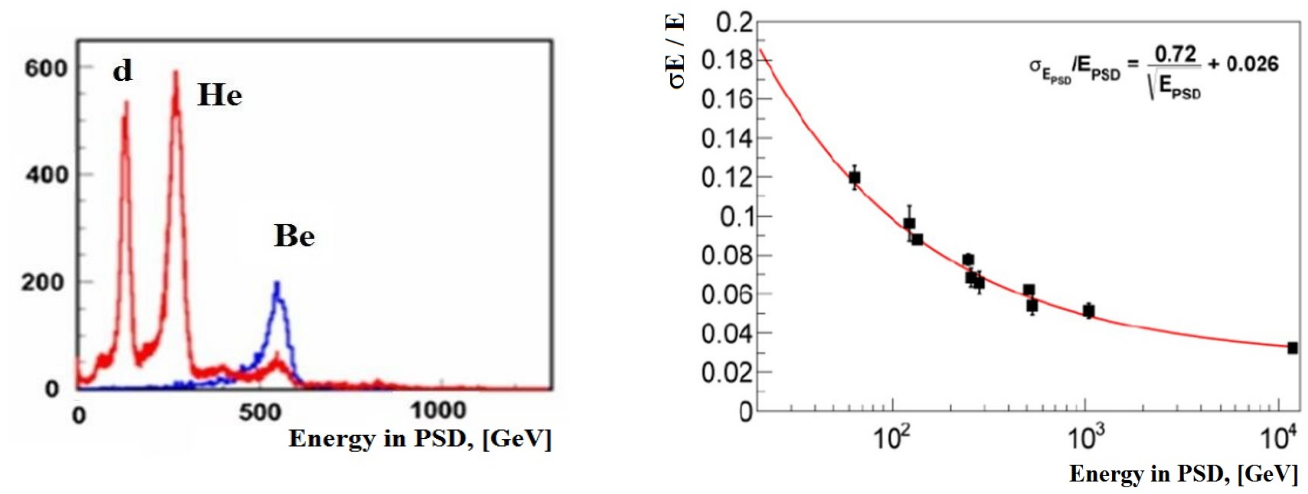

Fig.7. Left plot: reconstructed energy in the PSD for beam fragments of $75 \mathrm{AGeV}$ from the 2012-2013 Be+Be beam time period (left). Red color: all events, blue color: events selected as Be ions. Right plot: energy resolution for fragments in the Be beam.

front-end electronics (FEE) was studied. Be-ions were obtained by fragmenting a primary $\mathrm{Pb}$ beam. The calorimeter response is shown in Fig.7 (left) for beam fragments in the beamline set for the rigidity of $\mathrm{Be}$ ions at $75 \mathrm{AGeV}$ for all events (red) and events selected as Be ions with the A-detector designed for tagging different fragments. Fig.7 (right) shows the energy resolution measured with the PSD in events selected with the interaction trigger is shown in Fig.8. Various centrality classes are shown in color. 


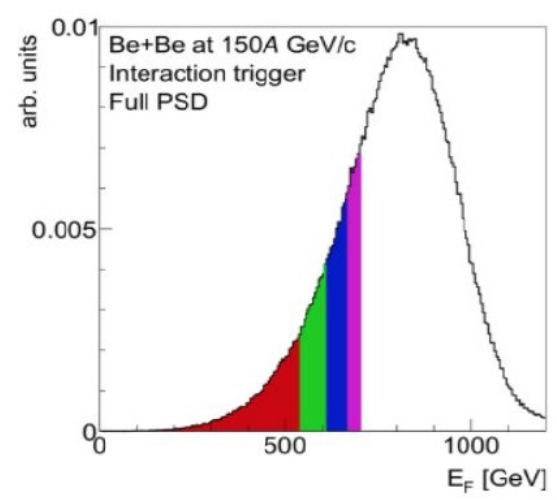

Fig.8. Distribution of forward energy $\left(\mathrm{E}_{\mathrm{F}}\right)$ measured with the PSD. Centrality classes are shown with different colors for bins from 0-5\% (red) till 15-20\% (magenta).

In $2015 \mathrm{Ar}+\mathrm{Sc}$ collisions were studied at beam momenta of 19, 30, 40, 75 and $150 \mathrm{AGeV} / \mathrm{c}$. The PSD was equipped with special "degraders" - brass cylinders of $5 \mathrm{~cm}$ diameter and $5 \mathrm{~cm}$ and $10 \mathrm{~cm}$ lengths - placed in front of the central modules. The degraders were used to break up argon ion before they reach the calorimeter surface. This results in spreading the energy of hadronic shower over the central modules of the calorimeter reducing light saturation effects in the sections. Two-dimension plots of track multiplicity form the TPCs versus energy $\mathrm{E}_{\mathrm{PSD}}$ measured by the PSD are shown in Fig.9 for Ar+Sc collisions at the used beam momenta.

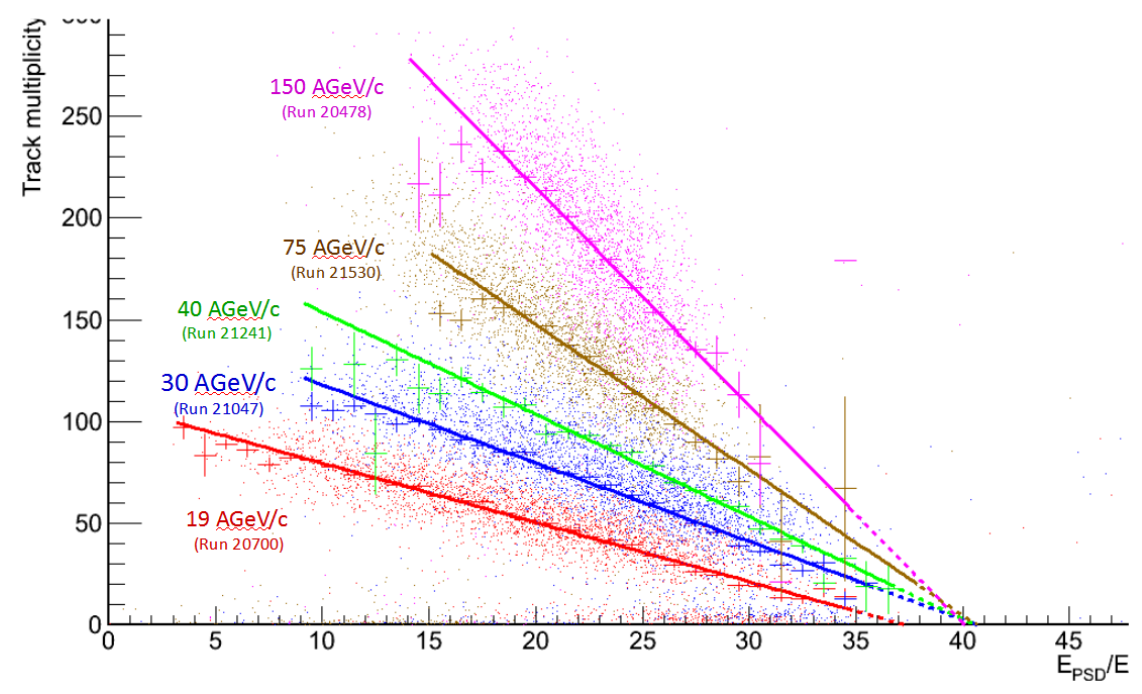

Fig.9. Track multiplicities from the TPCs versus scaled energy measured in the PSD calorimeter for the interaction trigger in Ar+Sc collisions recorded by NA61/SHINE in 2015. See text for details.

The energy $E_{\text {PSD }}$ was measured for events with centrality selection of about $0-30 \%$. Track multiplicities were calculated from the TPC chambers after full track reconstruction. Energies in the PSD were scaled to the beam energy per nucleon. Linear fits are extrapolated to the $\mathrm{X}$ axis crossing at about about the number of nucleons in argon ions (40) as expected. 
In 2016 first lead-lead collisions were recorded by NA61/SHINE at energies 30 and 150 AGeV. Results for the PSD performance are shown in Fig.10.
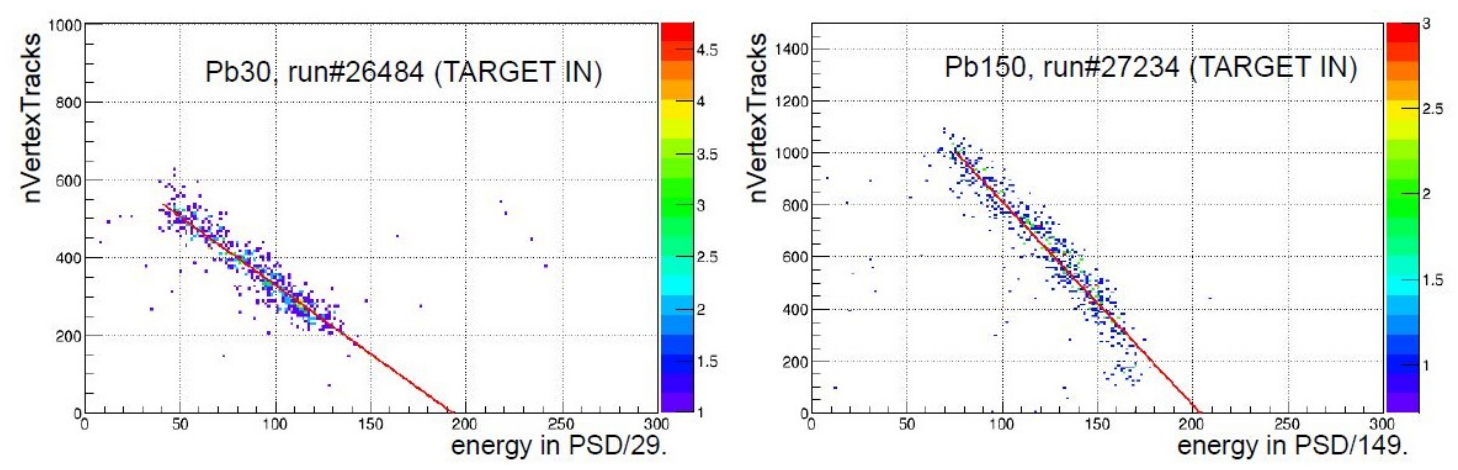

Fig.10. Track mutiplicity from the TPCs versus PSD energy for $\mathrm{Pb}+\mathrm{Pb}$ collisions recorded by NA61/SHINE in 2016.

Extrapolations from line fits cross the PSD energy axis at value $\sim 200$ which is close to the expected number of 208 for $\mathrm{Pb}$ ions. One can see the start of longitudinal shower leakage for $150 \mathrm{AGeV} \mathrm{Pb}+\mathrm{Pb}$ collision events with track multiplicities lower then 300 . For $\mathrm{Pb}$ ions the leakage is visible even for $30 \mathrm{AGeV}$. This is shown in Fig.11 where spectra from $\mathrm{Pb}$ ions and minimum biased events are compared.

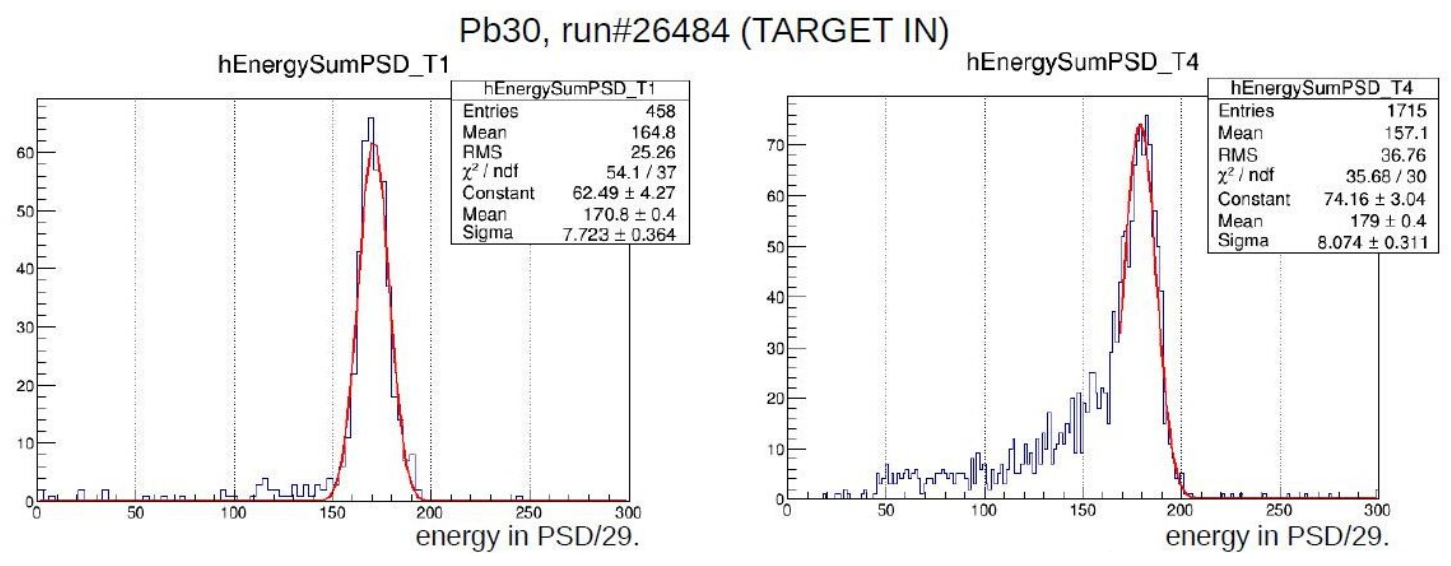

Fig.11. Response of the PSD for Pb ions for $30 \mathrm{AGeV}$ (left) and minimum biased events (right). Leakage of hadronic showers shifts the peak position down from the expected value of about 208. 

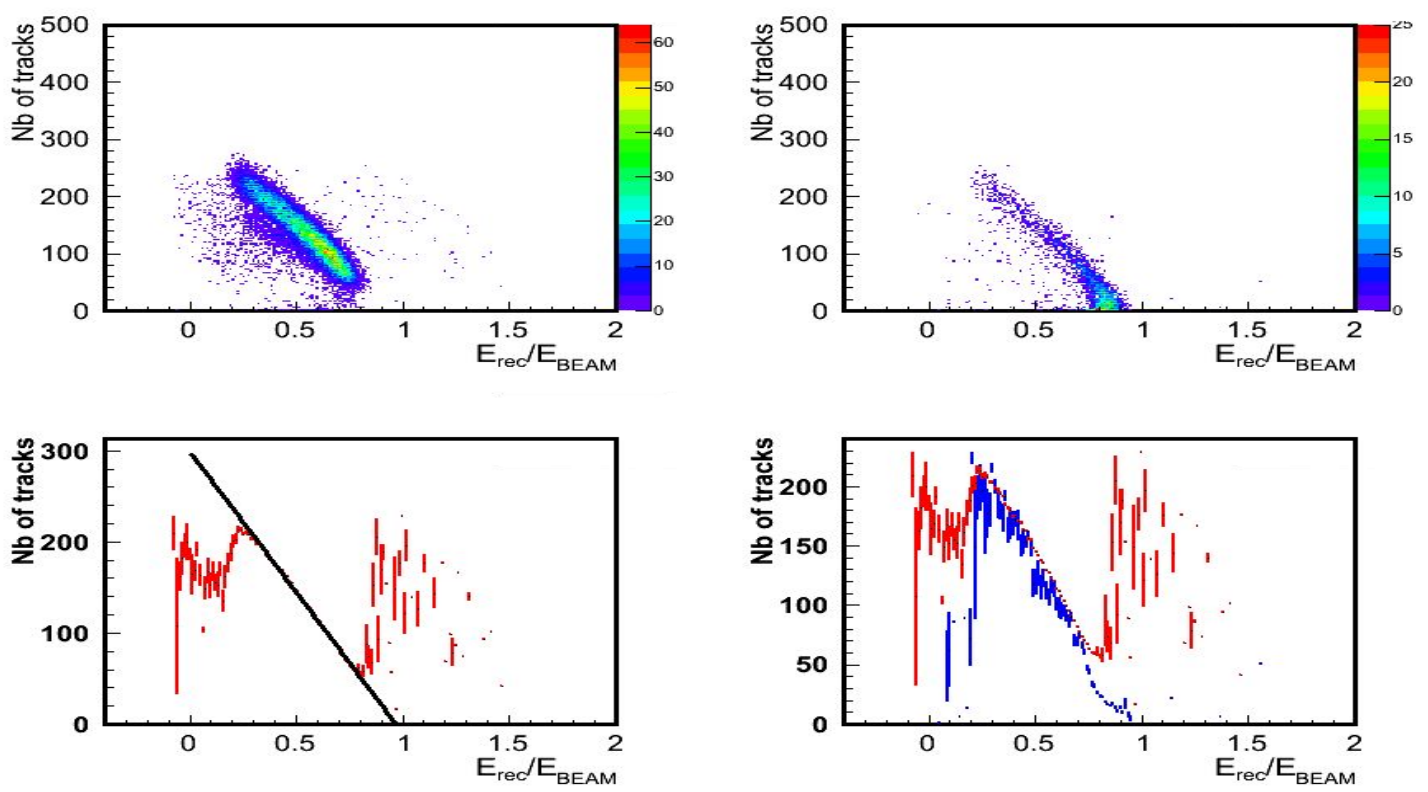

Fig.12. Performance results for Xe+La collision in 2017. Top row: multiplicity from the TPCs versus energy measured in the PSD at $30 \mathrm{AGeV}$.Bottom row: Fits to profile of $2 \mathrm{D}$ histograms and extrapolated to the $\mathrm{X}$ axis. Measured energies are normalized to the beam energy. Left (right) panels show results for interaction (minimum bias) triggered events.

The performance of the PSD in the $2017 \mathrm{Xe}+\mathrm{La}$ beam period of NA61/SHINE is presented in Fig.12. Here also the effects of shower leakage are visible for events with low track multiplicities in minimum bias triggered events.

\section{Calorimeter system upgrade the NA61/SHINE experiment beyond 2020.}

The program of the NA61/SHINE experiment has been extended to include open charm mesons measurements with a new and improved vertex detector. Such measurements were already started in 2017 and 2018 and results from $\mathrm{Xe}+\mathrm{La}$ and a small statistics sample of $\mathrm{Pb}+\mathrm{Pb}$ collisions are under analysis. The intensity of the $\mathrm{Pb}$ beam will be increased by one order of magnitude in order to meet the envisaged physics goals. This will cause radiation problems for PSD calorimeter and an upgrade of calorimeter system has become necessary. The radiation doses simulated with FLUKA are shown in Fig.13. 


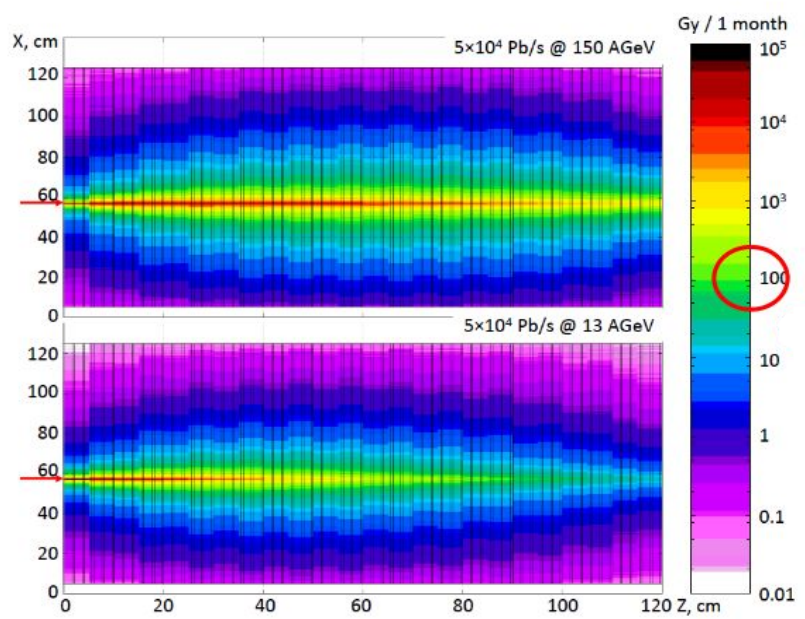

Fig.13. Radiation doses expected in high intensity beams of $\mathrm{Pb}$ at $150 \mathrm{AGeV}$ and $13 \mathrm{AGeV}$ in one month of current PSD operation (left). Red circle shows the level of maximum safe dose for scintillation plastic to keep its properties unchanged. Right plot: radial distributions of doses at a depth of $10 \mathrm{~cm}$ from the front face of the MSPD (green line) and the FPSD (blue line).

The simulations show that current version of the PSD hadron calorimeter will not survive in such radiation conditions. The new concept of the PSD calorimeter system is presented in Fig. 14. The proposed system will consist of two calorimeters: one main calorimeter (MPSD) of 32 modules with a beam hole in the center and a downstream forward calorimeter (FPSD) with $3 \times 3$ modules structure. The MPSD will measure interaction participants and light spectator fragments while the FPSD will be used for measuring heavy fragments and forming the interaction trigger based on event centrality. Radiation doses calculated for the MPSD + FPSD system are shown in Fig.15. For the central module of the FPSD the dose is still too high. The proposed solution is to make small holes in all scintillation plates in this module. In this case the

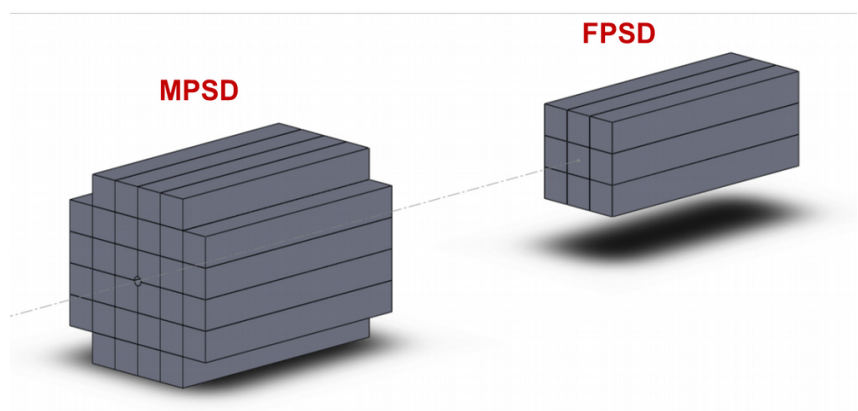

Fig.14. Proposed PSD calorimeter upgrade for NA61/SHINE beyond 2020.

performance of the calorimeter will be stable during a run period. A prototype of the future FPSD has been constructed and assembled in 2017 and was tested in the NA61/SHINE beam line in 2018. A view of the FPSD prototype is shown in Fig.16. 

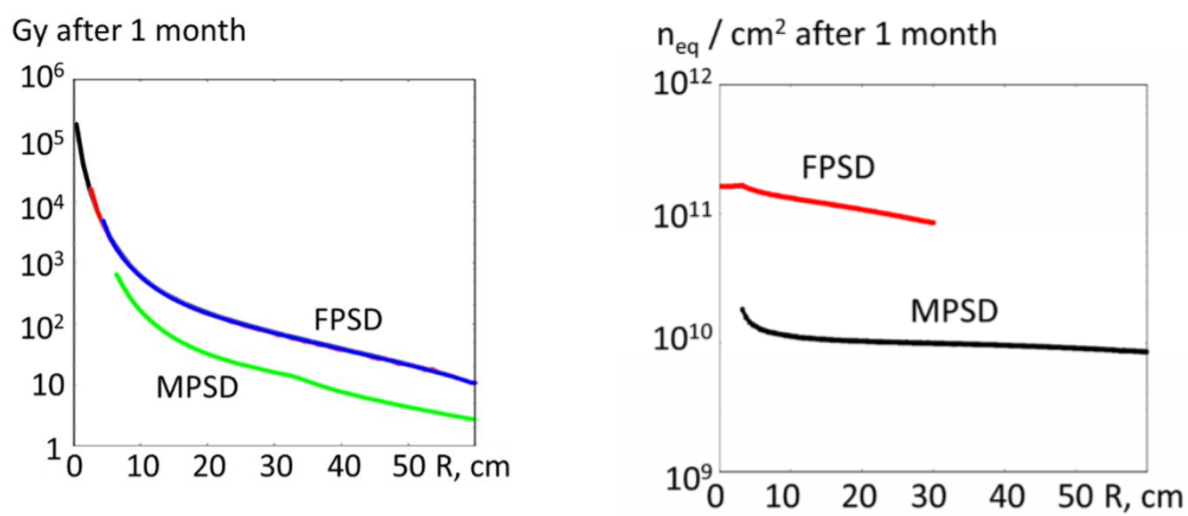

Fig.15. Radial distributions of doses from ionizing particles in the calorimeters at a depth of $10 \mathrm{~cm}$ from the front faces of the MPSD and the FPSD (left). Radial neutron fluence at the location of the MPPCs (right).



Fig.16. View of the current PSD (close position) and the prototype of the $3 \times 3$ modules forward hadron calorimeter FPSD (far position) installed for tests on the NA61/SHINE beamline.

\section{Conclusions}

The performance of the forward hadron calorimeter PSD of the NA61/SHINE experiment was presented. The energy resolution of the PSD for single protons can be described by $56.8 \%$ stochastic, $3.9 \%$ constant and $3.8 \mathrm{GeV}$ noise terms, respectively. The energy measured by the PSD for interaction trigger events in ion-ion collisions for $\mathrm{Be}+\mathrm{Be}, \mathrm{Ar}+\mathrm{Sc}, \mathrm{Xe}+\mathrm{La}$ and $\mathrm{Pb}+\mathrm{Pb}$ systems shows a good correlation with reconstructed track multiplicities obtained from the TPCs. Saturation in the readout of the light from hadronic showers is visible for minimum biased events as well as for ions hitting the calorimeter. An upgrade of the PSD is needed for the high beam intensities expected at NA61/SHINE facility beyond 2020. A two calorimeter system 
of MPSD + FPSD has been proposed. The first prototype of the FPSD has been constructed and tested in the NA61/SHINE beamline.

\section{Acknowledgments}

This work was partially supported by the Ministry of Science and Higher Education of the Russian Federation, grant N 3.3380.2017/4.7, and by the National Research Nuclear University $\mathrm{MEPhI}$ in the framework of the Russian Academic Excellence Project (contract No. 02.a03.21.0005, 27.08.2013).

\section{References}

[1] M. Gazdzicki and M. I. Gorenstein, Acta Phys. Polon. B 30, 2705 (1999) [hep-ph/9803462].

[2] N. Abgrall et al. (NA61 Collaboration), 2014, JINST 9, P06005, doi:10.1088/17480221/9/06/P06005.

[3] N. Abgrall et al. [NA61/SHINE Collaboration], Eur. Phys. J. C 74, no. 3, 2794 (2014) 222 doi:10.1140/epjc/s10052-014-2794-6 [arXiv:1310.2417 [hep-ex]].

[4] A. Aduszkiewicz et al. [NA61/SHINE Collaboration], Eur. Phys. J. C 76, no. 11, 635 (2016) 224 doi:10.1140/epjc/s10052-016-4450-9 [arXiv:1510.00163 [hep-ex]].

[5] A. Aduszkiewicz et al. [NA61/SHINE Collaboration], Eur. Phys. J. C 77, no. 2, 59 (2017) 226 doi:10.1140/epjc/s10052-017-4599-x [arXiv:1610.00482 [nucl-ex]]. 